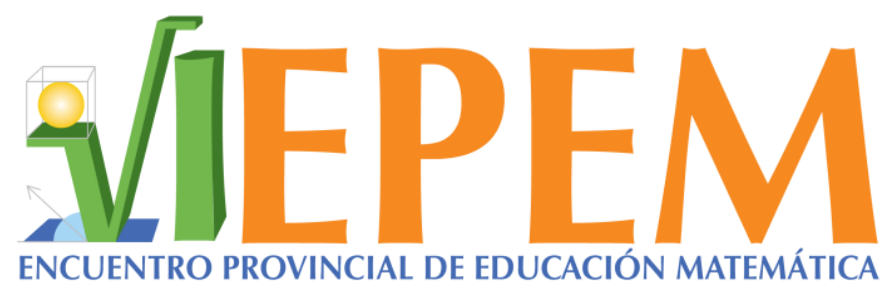

VI Encuentro Provincial de Educación Matemática.

27 al 29 de setiembre, 2017. Puntarenas, Costa Rica.

\title{
Conocimiento pedagógico que pone en práctica un profesor de Matemática al enseñar los conceptos básicos de función en Educación Secundaria
}

\begin{abstract}
Resumen
Este trabajo caracteriza el Conocimiento Pedagógico del Contenido utilizado por un profesor de Matemática en su práctica docente al enseñar los conceptos básicos de función. Se fundamenta en el modelo del Conocimiento Matemático para la Enseñanza (Hill, Ball y Schilling, 2008). Corresponde a una investigación cualitativa descriptiva basada en el estudio de casos. En particular, el conocimiento mostrado por el profesor se caracterizó por la utilización de estrategias para atender distintos estilos de aprendizaje, la adaptación de las tareas a los lineamientos curriculares y al nivel cognitivo del estudiante y por la variedad de caminos de aprendizaje utilizados y su adecuación en la resolución de tareas.
\end{abstract}

Jonathan Espinoza González

jonaespinoza@una.cr

Universidad Nacional

Costa Rica

Miguel Picado Alfaro

miguel.picado.alfaro@una.cr

Universidad Nacional

Costa Rica

Palabras clave: Conocimiento pedagógico del contenido; secundaria; funciones; enseñanza.

\section{Introducción}

El estudio de la práctica y el desempeño del docente de Matemática en el aula podría generar insumos para realizar cambios en la actividad pedagógica. Por ejemplo, investigaciones recientes sobre el conocimiento matemático señalan la importancia que tiene una buena planificación matemática al momento de enseñar conceptos matemáticos.

Ponencia

Espinoza, J. y Picado, M. (2017). Conocimiento pedagógico que pone en práctica un profesor de Matemática al enseñar los conceptos básicos de función en Educación Secundaria. En Y. MoralesLópez, M. Picado, R. Gamboa, C. Martínez, M. Castillo y R. Hidalgo (Eds.), Memorias del VI Encuentro Provincial de Educación Matemática, Costa Rica, 2017 (pp. 55-65). Heredia: Universidad Nacional. ISBN: 978-9968-9661-5-3. DOI: http://dx.doi.org/10.15359/epem.6.15 
En este sentido, un docente que posee un fuerte conocimiento matemático tiene más herramientas para ayudar a sus estudiantes a comprender en profundidad el tema (Haciomeroglu, 2006).

Por otra parte, González (1999) indica que es común escuchar comentarios de estudiantes relacionados con el desempeño del docente en el aula, en cuanto a que el profesor posee mucho conocimiento matemático, pero que tiene dificultades para enseñarlo. Este tipo de aseveraciones muestra que no es suficiente con conocer la disciplina, sino que se debe profundizar más en su dominio, contenido y enseñanza. Un camino que se puede emprender con el estudio del conocimiento pedagógico del contenido.

El conocimiento pedagógico del contenido refiere a la variedad de conocimientos sobre la enseñanza que todo profesor debe dominar sobre un tema específico; este genera mayor respeto por la profesión de la enseñanza y cubre un vacío sobre el conocimiento del profesor (Pinto y González, 2006). También es un elemento que caracteriza a los buenos profesores (Shulman, 2005).

En Costa Rica, el Ministerio de Educación Pública (MEP), realizó en el año 2010 un examen diagnóstico para evaluar el conocimiento de conceptos matemáticos de los profesores de Matemática que trabajan en instituciones públicas de Educación Secundaria. Los resultados indican que $43,3 \%$ de estos docentes muestra limitaciones en cuanto al dominio de los contenidos matemáticos que se enseñan en Educación Secundaria (Programa Estado de la Nación en Desarrollo Humano Sostenible, 2013). Esta problemática en particular podría influir en la enseñanza de los conceptos matemáticos y en el rendimiento académico de los estudiantes.

En el caso de los estudiantes, los resultados de las pruebas de bachillerato muestran la dificultad que tienen en esta materia (MEP, 2012). En particular, el análisis realizado a los resultados de la prueba aplicada en el año 2013 revela que dentro de los contenidos con menor porcentaje de aprobación son aquellos vinculados con el tema de Funciones, la mayoría menor al 50\% (MEP 2014); esto evidencia la existencia de posibles errores y dificultades en el aprendizaje de estos conceptos y procedimientos particulares.

Este estudio enfoca al profesor como un agente responsable de los procesos de enseñanza y aprendizaje de la Matemática. Caracteriza el Conocimiento Pedagógico del Contenido (CPC) de un docente de Matemática al enseñar los conceptos básicos de función en cuarto año de Educación Secundaria. En general, destaca la importancia de poseer un conocimiento pedagógico específico para la Matemática que permita mejorar la práctica docente, especialmente en aquellos temas con mayor dificultad para los estudiantes.

\section{Marco teórico}

La fundamentación teórica de esta investigación se basa en las ideas propuestas inicialmente por Shulman $(1986,1987)$ sobre el conocimiento profesional del profesor de Matemática y desarrolladas posteriormente por Ball y colaboradores (Ball, Hill y Bass, 2005; Ball, Thames y Phelps, 2008; Hill, Ball y Schilling, 2008). También se emplea el análisis didáctico (Gómez, 2007) con el propósito de determinar indicadores que permitan describir el conocimiento del profesor. 
En sus estudios, Shulman $(1986,1987)$ destaca las diferencias entre el conocimiento del contenido y el conocimiento del contenido para la enseñanza. El primero permite tener dominio de las definiciones y poder justificar proposiciones, así como conocer la aplicación de los contenidos en otras disciplinas (Shulman, 1986), mientras que el conocimiento del contenido para la enseñanza se refiere a una pedagogía específica para la Matemática (Shulman, 1987), mediante la cual se presenta el contenido matemático de la mejor manera posible.

Por su parte, Hill, Ball y Schilling (2008), consideran que los docentes de Matemática necesitan un conocimiento específico para enseñar la materia que va más allá del conocimiento matemático, a este lo llaman Conocimiento Matemático para la Enseñanza (CME) y lo definen como "el conocimiento matemático que los profesores utilizan en el aula para producir aprendizaje y crecimiento en los alumnos" (p. 374).

A partir de la propuesta de Shulman (1986, 1987), Ball, Thames y Phelps (2008) proponen un modelo para el CME que distingue entre dos dominios de conocimiento: el Conocimiento del Contenido Matemático (CCM) y el Conocimiento Pedagógico del contenido (CPC). Además, establecen tres subdominios para cada uno de los dominios descritos. En la siguiente figura 1 se muestra este modelo.

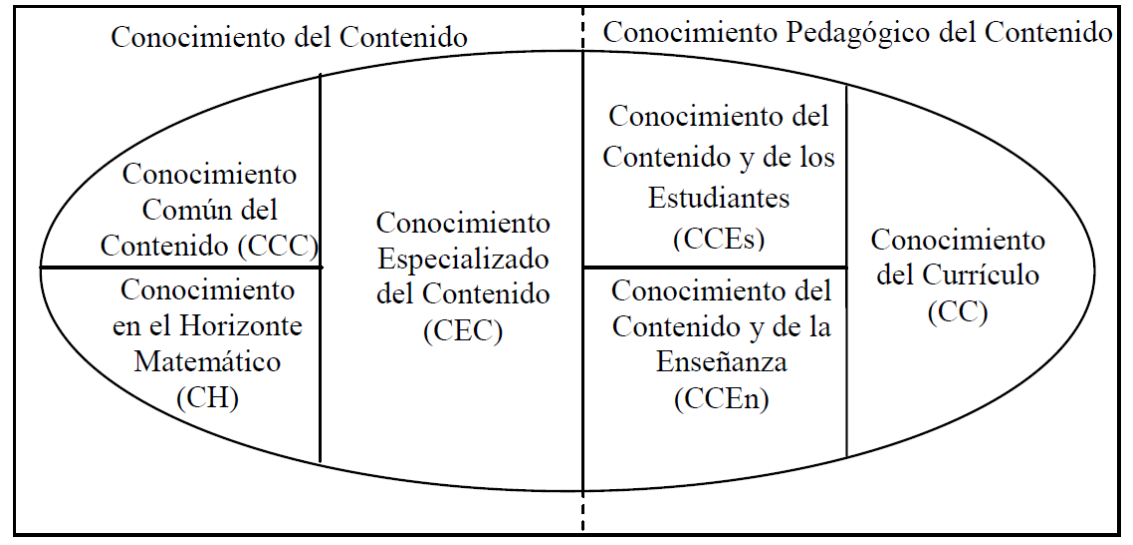

Figura 1. Dominios de conocimiento matemático para la enseñanza (Ball, Thames y Phelps, 2008). Extraído de Content Knowledge for Teaching: What Makes It Special? Journal of Teacher Education, 59(5), 389-407.

Como muestra la figura 1, el CCM — que enfatiza las matemáticas- está integrado por tres subdominios: el Conocimiento Común del Contenido (CCC), el Conocimiento en el Horizonte Matemático (CHM) y el Conocimiento Especializado del Contenido (CEC). Para una mayor caracterización de estos componentes pueden consultarse los trabajos de Ball, Thames y Phelps (2008) y Rojas (2010).

Por otra parte, el CPC, que enfoca los procesos de enseñanza y aprendizaje de las matemáticas, se compone por los subdominios: el Conocimiento del Contenido y de los estudiantes (CCEs) que corresponde a un conocimiento particular del contenido Matemático que permite determinar cuánto saben y cómo piensan y aprenden los estudiantes ese contenido; el Conocimiento del Contenido y la Enseñanza (CCEn) que se 
refiere al conocimiento que entrelaza el conocimiento de un contenido matemático particular y la enseñanza del mismo; y el Conocimiento del currículo (CC) que alude al conocimiento de las disposiciones curriculares vigentes.

En este sentido, un docente que sobresale por su conocimiento del CPC anticipa los principales errores y las dificultades más comunes que presentan los estudiantes al aprender un contenido matemático (CCEs), además conoce sobre diversos recursos, materiales y estrategias para enseñar un tema (CCEn) y también domina las orientaciones curriculares relacionadas con los objetivos, contenidos, fines que permiten al docente guiar su práctica y seleccionar las tareas adecuadas al nivel educativo de los estudiantes (CC).

Además de la teoría descrita sobre el conocimiento matemático para la enseñanza, se empleó el análisis didáctico (Gómez, 2007), entendido este como un "procedimiento con el que es posible explorar, profundizar y trabajar con los diferentes y múltiples significados del contenido matemático escolar, para efectos de diseñar, llevar a la práctica y evaluar actividades de enseñanza y aprendizaje” (p. 18-19). El análisis didáctico se compone por cinco análisis parciales: análisis conceptual, de contenido, cognitivo, de instrucción y de evaluación.

El análisis conceptual aborda la diversidad de significados de un concepto matemático y el análisis de contenido enfatiza la estructura conceptual, las aplicaciones y representaciones de los conceptos matemáticos. El análisis cognitivo enfoca el aprendizaje de las matemáticas por parte de los estudiantes e incluye las expectativas (objetivos), las limitaciones (errores y dificultades) y las oportunidades (tareas) de aprendizaje. El análisis de instrucción analiza el diseño, selección y la secuencia en que se presentarán las tareas en la unidad didáctica. Por último, el análisis de evaluación describe la comprensión de los estudiantes respecto al tema desarrollado según las estrategias utilizadas y las tareas resueltas.

Para identificar el conocimiento que pone en práctica un profesor al enseñar los conceptos de función se emplea el análisis de contenido, cognitivo y de instrucción desde la perspectiva de Rojas, Flores y Ramos (2013) quienes combinan estos análisis parciales con el Conocimiento Matemático para la Enseñanza.

\section{Metodología}

El abordaje metodológico de la investigación corresponde a un estudio de carácter cualitativo descriptivo basado en los estudios de casos (McMillan y Schumacher, 2005).

La fuente de información ha sido un docente de matemática de educación secundaria en servicio, seleccionado a partir de criterios técnicos definidos según los propósitos de la investigación, así como criterios para identificar un profesor experto (Rojas, Carrillo y Flores, 2012), entre ellos: tener al menos cinco años de experiencia como docente de Educación Secundaria, haber enseñado el tema de funciones al menos una ocasión, ser recomendado por directivos institucionales y autoridades educativas y tener disponibilidad para participar en el estudio. 
Los datos se recolectaron por medio de la observación no participante. En total se observaron cinco sesiones de clase (de 40 minutos u 80 minutos) que fueron registradas en audio y video, esto con el aval de los encargados legales de los estudiantes, el profesor y las autoridades institucionales donde se realizó el estudio. En total se cuenta con cerca de 200 minutos de grabación de audio y video. La descripción de cada episodio de clase se encuentra en el siguiente apartado.

\section{Categorías de análisis}

Para el análisis de la información se adaptaron las categorías sobre el CME implementadas por Rojas, Flores y Ramos (2013). Para cada categoría se diseñó una serie de unidades de análisis para estudiar con particularidad el CME de un profesor cuando enseña los conceptos básicos de función. A continuación, se muestran únicamente las categorías asociadas a los subdominios del CPC, esto debido al foco de atención del trabajo que se presenta y a la gran cantidad de unidades de análisis asociadas a estas categorías.

\section{Tabla 1}

Categorías para cada uno de los subdominios del Conocimiento Pedagógico del Contenido

\begin{tabular}{l|l}
\hline Subdominio & Categorías \\
\hline CCEs & Referencia a los conocimientos esperados que aprendan los estudiantes, teniendo \\
& presente el nivel educativo y los conocimientos previos de los que parten. \\
& Dominio de las características de aprendizaje del contenido matemático. \\
- Alusiones a los errores y las dificultades que los estudiantes pueden presentar en \\
la resolución de las tareas propuestas, en el empleo de conceptos o en la \\
aplicación de procedimientos. \\
- Actividades o preguntas planteadas a los estudiantes para prever y vencer las \\
dificultades y corregir los errores posibles al estudiar el contenido matemático. \\
- Asignación de tareas que refuercen conceptos o procedimientos matemáticos, \\
relacionados con las dificultades y errores de aprendizaje de los estudiantes. \\
- Actividades o preguntas planteadas a los estudiantes para vencer las dificultades y \\
corregir errores detectados al estudiar el contenido matemático. \\
- Grado en que las tareas propuestas se adaptan a las finalidades de aprendizaje. \\
- Grado en que las tareas planteadas sean adecuadas al nivel escolar y cognitivo de \\
los estudiantes. \\
Variedad de tareas planteadas en relación con la diversidad del alumnado.
\end{tabular}




\begin{tabular}{l|l}
\hline CCEn & Adecuación entre los sistemas de representación empleados para enseñar las ideas \\
matemáticas específicas. \\
- Paralelismo entre las habilidades específicas planteadas (implícita o \\
explícitamente) y el desarrollo de la instrucción. \\
- Adecuación de las tareas propuestas para cada objetivo. \\
- Variedad de sistemas de representación que se emplean en las tareas planteadas. \\
- matemáticos. \\
- Evidencia de que el profesor dispone de un esquema de instrucción. \\
- Adecuación entre las tareas presentadas y los caminos de aprendizaje que se \\
intuyen. \\
- Riqueza de materiales y recursos didácticos empleados. \\
Adecuación de los recursos y materiales según el nivel de enseñanza y las \\
finalidades previstas de adquisición de conceptos y propiedades. \\
- Relación entre acciones que permiten el empleo de recursos y situaciones, que \\
envuelvan diversos significados y contextos. \\
- Grado en que los contenidos enseñados corresponden con los establecidos por el \\
currículo escolar de referencia o documentos oficiales que atienden al proceso de \\
enseñanza. \\
GC
\end{tabular}

\section{Análisis y discusión}

Para iniciar este apartado se muestra una descripción general de cada uno de los episodios observados. Seguidamente, se presentan los principales resultados obtenidos para cada uno de los subdominios del CPC.

\section{Descripción general de los episodios}

En el primer episodio se presenta a los estudiantes un problema sobre el costo y producción de artículos, esto para introducir el concepto de función. Para su resolución la clase se organiza en subgrupos de trabajo, mientras el profesor atiende consultas en cada subgrupo para finalmente resolver la tarea en la pizarra a partir de los aportes de los estudiantes.

En el segundo episodio se retoma el problema propuesto en el episodio anterior para recalcar los conceptos abordados (relación, función, conjunto, correspondencia y plano cartesiano) e introducir nuevos conceptos (preimagen, imagen, dominio, codominio y ámbito).

En el tercer episodio se selecciona una tarea que implica el trazo de una gráfica para reconocimiento del dominio y ámbito de una función. Luego, asigna dos tareas a los 
estudiantes para que sean resueltas en subgrupos con la atención de consultas por parte del profesor, posteriormente son revisadas en la pizarra.

En el cuarto episodio se presentan una serie de tareas para reconocer a partir de representaciones gráficas el dominio y rango de algunas funciones. El trabajo de los estudiantes es individual y el profesor atiende dudas.

En el quinto episodio se continua con la presentación de representaciones gráficas de funciones para identificar imágenes y preimágenes.

\section{Sobre el conocimiento del contenido y de los estudiantes (CCEs)}

La utilización de conocimientos previos se realiza en las últimas sesiones de clase cuando se introducen los conceptos de dominio y ámbito. No se logró observar alguna referencia a los conocimientos que se espera que aprendan los estudiantes, excepto en la sesión en que se introdujo el concepto de función.

Se logró constatar que el docente reconoce distintos estilos de aprendizaje de sus estudiantes, esto porque en la resolución de tareas, tanto por parte de los estudiantes como del profesor, se toman en cuenta aspectos del aprendizaje visual (principalmente mediante representaciones gráficas), auditivo (por medio de expresiones verbales), kinestésico y de lecto/escritura.

Solo en la primera sesión de clase el docente menciona posibles errores que se pueden presentar en la resolución de una tarea específica. Además, en ninguna de las actividades desarrolladas se aprecia una planificación previa tanto para detectar errores como para vencerlos. Los errores abordados son aquellos que surgen de forma espontánea durante la resolución de una tarea. Cabe destacar que durante la resolución de algunas tareas fue posible reconocer momentos en los que se podrían abordar algunos errores, pero el profesor no los aprovechó. Además, la asignación de tareas adicionales para reforzar conceptos o procedimientos matemáticos es escasa a lo largo de los episodios.

En relación con las dificultades, estas se presentan principalmente al momento de comprender los enunciados de las tareas y al identificar elementos en las representaciones gráficas. Estas y otras dificultades son afrontadas por el docente durante el trabajo individual o grupal mediante explicaciones que consideran los distintos estilos de aprendizaje. Además, no se observó alguna evidencia relacionada con un plan preliminar para abordar alguna dificultad.

Las finalidades pretendidas en las tareas presentadas se adaptan de forma adecuada con los lineamientos curriculares y al nivel escolar de los estudiantes, sin embargo, algunas de ellas no fueron aprovechadas al máximo para presentar diversos conceptos, por lo que se deduce que el docente planifica cada tarea para un concepto básico de función específico. Además, las tareas mostradas corresponden, casi en su totalidad, al nivel cognitivo de reproducción, siendo las más frecuentas las que corresponden a situaciones públicas y educativas y a contextos de carácter hipotético o auténtico. Por último, es escasa la utilización de tareas adicionales para reforzar conceptos o procedimientos matemáticos, excepto las empleadas para reforzar los conceptos de dominio y ámbito de una función. 


\section{Sobre el conocimiento del contenido y la enseñanza (CCEn)}

Las representaciones más frecuentes son las de tipo verbal, gráfico, simbóliconumérico y simbólico algebraico. En relación con las representaciones se observó la falta de precisión con la que fueron elaboradas (no se emplea una escala numérica apropiada), por lo que algunas de estas podrían provocar errores, dificultades o ambigüedades entre los estudiantes.

Las habilidades específicas propuestas en el plan de estudios se vinculan apropiadamente con la instrucción realizada. Además, es posible asociar cada tarea con alguna de las habilidades cognitivas propuestas en el programa de estudios, sin embargo, no fue posible identificar tareas que abordaran las habilidades de distinguir entre cantidades constantes y variables e identificar si una relación dada en forma tabular, simbólica o gráfica corresponde a una función. Esto está relacionado con la falta de representaciones tabulares durante las lecciones y con el uso de representaciones gráficas y símbolos para identificar conceptos básicos de función.

Un aspecto que sobresale de los episodios observados es la variedad de sistemas de representación empleadas por el docente, siendo el verbal el que se presenta con mayor frecuencia, seguido por las representaciones simbólicas de tipo algebraico y numérico, y las gráficas.

Las tareas empleadas para reforzar conceptos matemáticos mantienen su caracterización. Son tareas de reproducción, de carácter público y educativo, auténticas o hipotéticas.

Durante las lecciones ha sido posible reconocer un esquema de instrucción en el que predomina el uso de la clase magistral. El profesor capta la atención de los estudiantes, esto a pesar de distractores que se presentan en las sesiones (partidos del mundial de fútbol); comunica las habilidades pretendidas cuando corresponde y alude a conocimientos previos en caso de ser necesario; presenta contenidos guiando el aprendizaje y provocando el desempeño en una parte considerable del grupo de estudiantes. Además, realiza preguntas a sus estudiantes de forma constante para valorar el nivel de aprendizaje.

Las tareas presentadas se caracterizan por considerar al menos un camino de aprendizaje en los que es posible reconocer las capacidades que se pretenden lograr o reforzar. Así mismo, se logró determinar que el docente utiliza una variedad de caminos de aprendizaje los cuales adecúa según sea la tarea a resolver, no obstante, en general, estos caminos no consideraban el reconocimiento de posibles errores. Únicamente en el segundo episodio de clase no se reconoce un camino de aprendizaje dado que la tarea mostrada ya estaba resuelta y no en proceso de resolución.

Los recursos didácticos utilizados son la pizarra y regla (metro), esta última principalmente para trazar la gráfica de una función mediante la cual explica a los estudiantes conceptos como dominio, ámbito, imagen y preimagen, sin aprovecharla (la gráfica) para mostrar las relaciones entre estos conceptos o la diversidad de significados de los mismos. En relación con los materiales didácticos, el docente emplea un libro (folleto) de su autoría el cual incluye, además de las definiciones de los contenidos asociados a las funciones, tareas que se emplean para reforzar conceptos como dominio, ámbito, imagen y preimagen. 


\section{Sobre el conocimiento del currículo (CC)}

Todos los conocimientos propuestos en el programa de estudios son abordados por el profesor durante las cinco sesiones de clase observadas. Se detectaron algunos conocimientos vinculados al tema de funciones que no forman parte del currículo pero que refuerzan los conceptos mostrados. En relación con el significado dado a los conceptos básicos de función y las tareas presentadas sobre estos, se observó que en general corresponden a niveles básicos de aprendizaje.

Las tareas presentadas carecen de un vínculo con los ejes curriculares sobre el uso de la historia de la matemática y la tecnología. Tampoco se identifica explícitamente una atención a las creencias y actitudes positivas hacia las matemáticas. Por su parte, las recomendaciones sobre la resolución de problemas y la contextualización se presentan sin llegar a ser un punto fuerte del plan de clase.

Por último, los recursos y materiales empleados se adecúan a las orientaciones metodológicas e indicaciones puntuales propuestas en el plan de estudios.

\section{Consideraciones finales}

Las categorías y unidades de análisis utilizadas permiten reconocer indicadores sobre el conocimiento pedagógico de un profesor de Matemática cuando enseña conceptos matemáticos, en este caso conceptos básicos de función.

El análisis realizado proporciona descriptores que fomentan la reflexión sobre la práctica docente desde un marco específico que acentúa elementos relacionados con el aprendizaje y enseñanza de contenidos matemáticos particulares y de los lineamientos curriculares que orientan estos procesos. Por ejemplo, la atención previa de errores o el abordaje de aquellos que puedan surgir durante la lección y la selección apropiada de tareas.

\section{Reconocimiento}

Esta contribución es producto del Proyecto SIA 0127-13 titulado Conocimiento matemático para la enseñanza de los profesores sobre el tema de funciones en el Ciclo Diversificado de la Educación Matemática en Costa Rica desarrollado en la Escuela de Matemática de la Universidad Nacional de Costa Rica.

\section{Referencias}

Ball, D., Hill, H. y Bass, H. (2005). Knowing mathematics for teaching: Who knows mathematics well enough to teach third grade, and how can we decide? American Educator, 29(1), 14-17, 20-22, 43-46. Recuperado de http://hdl.handle.net/2027.42/65072 
Ball, D., Thames, M. y Phelps, G. (2008). Content Knowledge for Teaching: What Makes It Special? Journal of Teacher Education, 59(5), 389-407. Recuperado de https://doi.org/10.1177/0022487108324554

Gómez, P. (2007). Desarrollo del conocimiento didáctico en un plan de formación inicial de profesores de matemáticas de secundaria (Tesis doctoral). Universidad de Granada, España.

González, F. (1999). Los nuevos roles del profesor de matemática: retos de la formación de docentes para el siglo XXI. En R. M. Farfán, C. E. Matías, D. Sánchez y Á. Tavarez (Eds.), Conferencia inaugural de la Decimotercera Reunión Latinoamericana de Matemática Educativa: Relme 13 (pp. 1-27). Santo Domingo, República Dominicana: Grupo Editorial Iberoamérica S.A.

Haciomeroglu, G. (2006). Prospective secondary teachers' subject matter knowledge and pedagogical content knowledge of the concept of function (Tesis doctoral). The Florida State University College of Education, Estados Unidos de América.

Hill, H., Ball, D. y Schilling, S. (2008). Unpacking Pedagogical Content Knowledge: Conceptualizing and Measuring Teacher's Topic-Specific Knowledge of Students. Journal for Research in Mathematics Education, 39(4), 372-400. Recuperado de http://www.nctm.org/Publications/journal-for-research-in-mathematicseducation/2008/Vol39/Issue4/Unpacking-Pedagogical-Content-Knowledge_Conceptualizing-and-Measuring-Teachers_-Topic-Specific-Knowledge-of-Students/

McMillan, J. y Schumacher, S. (2005). Investigación educativa (5ª . ed). Madrid, España: Pearson Educación.

Ministerio de Educación Pública. (2012). Informe nacional 2011. Resultados de las pruebas nacionales de bachillerato de la educación formal. San José, Costa Rica: Dirección de Gestión y Evaluación de la Calidad.

Ministerio de Educación Pública. (2014). Informe nacional 2013. Resultados de las pruebas nacionales de bachillerato de la Educación Formal. San José, Costa Rica: Dirección de Gestión y Evaluación de la Calidad.

Pinto, J. E. y González, M. T. (2006). Sobre la naturaleza conceptual y metodológica del conocimiento del contenido pedagógico en Matemáticas: una aproximación para su estudio. En M. Bolea, M. Moreno y M. J. González (Eds.), Investigación en educación matemática: Actas del X Simposio de la Sociedad Española de Investigación en Educación Matemática (pp. 237-255). Huesca, España: Instituto de Estudios Altoaragoneses. Recuperado de http://funes.uniandes.edu.co/1293/

Programa Estado de la Nación en Desarrollo Humano Sostenible. (2013). Cuarto Informe del Estado de la Educación: Desempeño de la Educación General Básica y el ciclo diversificado en Costa Rica. Informe final. Recuperado de http://estadonacion.or.cr/files/biblioteca_virtual/educacion/004/castro_desempeno-edbasica-y-diversificado.pdf

Rojas, N. (2010). Conocimiento para la enseñanza y calidad matemática de la instrucción del concepto de fracción: estudio de caso de un profesor chileno. Tesis de maestría, Universidad de Granada, España.

Rojas, N., Carrillo, J. y Flores, P. (2012). Características para identificar a profesores de matemáticas expertos. En Estepa, A., Contreras, Á., Deulofeu, J., Penalva, M., García, F. y Ordoñez, L. (Eds.), Investigación en Educación Matemática XVII (pp. 79-485). Jaén, España: SEIEM. 
Rojas, N., Flores, P. y Ramos, E. (2013). El análisis didáctico como herramienta para identificar conocimiento matemático para la enseñanza en la práctica. En L. Rico, J. L. Lupiáñez y M. Molina (Eds.), Análisis didáctico en educación matemática. Metodología de investigación, innovación curricular y formación de profesores (pp. 192-208). Granada, España: Comares.

Shulman, L. S. (1986). Those who understand: Knowledge growth in teaching. Educational Researcher, 15(2), 4-14.

Shulman, L. S. (1987). Knowledge and Teaching. Foundations of the New Reform. Harvard Educational Review, 57(1), spring 1987 Issue, 1-23.

Shulman, L. S. (2005). Conocimiento y enseñanza: Fundamentos de la nueva reforma. Revista de currículum y formación del profesorado, 9(2), 1-30.

\section{(c) $(7)(\mathrm{E})$}

Esta obra está bajo una licencia de Creative Commons Reconocimiento-NoComercialSinObraDerivada 4.0 Internacional. 